\title{
Radiation dose and cancer risk among pediatric patients undergoing interventional neuroradiology procedures
}

\begin{abstract}
Background: During interventional neuroradiology procedures, patients can be exposed to moderate to high levels of radiation. Special considerations are required to protect children, who are generally more sensitive to the short- and long-term detrimental effects of radiation exposure. Estimates of dose to the skin of children from certain interventional procedures have been published elsewhere, but we are not aware of data on dose to the brain or on the long-term risk of cancer from brain radiation. Objectives: Our goals were to estimate radiation doses to the brain in 50 pediatric patients who had undergone cerebral embolization and to assess their lifetime risks of developing radiation-related brain cancer. Materials and methods: Entrance-peak skin dose and various assumptions on conditions of exposure were used as input for dosimetric calculations to estimate the spatial pattern of dose within the brain and the average dose to the whole brain for each child. The average dose and the age of the child at time of exposure were used to estimate the lifetime risk of developing radiation-related brain cancer. Results: Among the 50 patients, average radiation doses to the brain were estimated to vary from $100 \mathrm{mGy}$ to
\end{abstract}

I. Thierry-Chef · S. L. Simon

Radiation Epidemiology Branch,

Division of Epidemiology and Genetics,

National Cancer Institute,

Rockville, MD 20892, USA

D. L. Miller

Department of Radiology and Radiological Sciences,

Uniformed Services University,

Bethesda, MD 20814, USA

Present address:

D. L. Miller

Department of Radiology, Albert Einstein Medical Center,

Philadelphia, PA 19141, USA

I. Thierry-Chef $(\bowtie)$

18 Domaine de la côte,

69530 Brignais, France

e-mail: thierrychef@hotmail.com

Tel.: +33-6-83830994
$1,300 \mathrm{mGy}$ if exposed to non-collimated fields and from $20 \mathrm{mGy}$ to $160 \mathrm{mGy}$ for collimated, moving fields. The lifetime risk of developing brain cancer was estimated to be increased by $2 \%$ to $80 \%$ as a result of the exposure. Given the very small lifetime background risk of brain tumor, the excess number of cases will be small even though the relative increase might be as high as $80 \%$. Conclusion: ALARA principles of collimation and dose optimization are the most effective means to minimize the risk of future radiation-related cancer.

Keywords Brain radiation exposure $\cdot$ Neurointerventional procedures $\cdot$ Lifetime risk radiation-induced carcinogenesis

\section{Introduction}

Interventional radiology procedures are potentially lifesaving and often necessary to correct certain medical conditions. Compared to conventional surgery, interventional procedures have certain benefits to patients, primarily decreased morbidity and quicker recovery times, since incisions are usually small and tissue injury is limited.

Most interventional procedures are performed in the heart, but the number of complex procedures performed on other organs has increased in recent years. Complex interventions often lead to higher radiation exposures. The medical and radiation protection communities have been concerned about the level of dose to the skin and organs of patients and the associated risk of developing radiationrelated cancer at a later date. For that reason, the RAD-IR study [1-3] was carried out between 1998 and 2001 in seven medical centers in the US to assess doses to the skin of patients involved in interventional procedures. Out of more than 2,000 procedures evaluated, 85 ( $4 \%$ of the total) were carried out on children between birth and 18 years of age. Among those, a group of 50 patients underwent interventional neuroradiology procedures to embolize intracranial aneurysms, arteriovenous malformations, or tumors. 


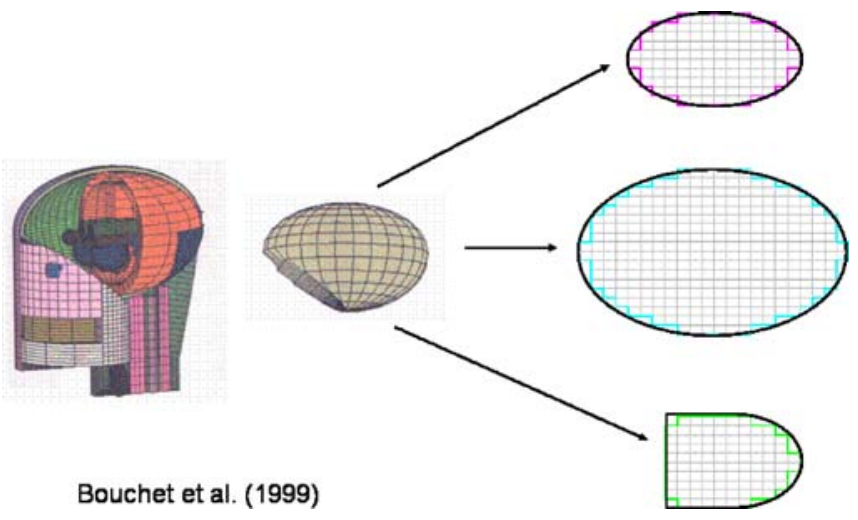

Fig. 1 Model used for dose calculation

Because interventional neuroradiology procedures are rarely carried out on pediatric patients, there is little information on doses to the brain from these procedures in children. Thus, estimating radiation dose was one important objective of the present study. Because children are more sensitive to the harmful effects of radiation and because they have a longer remaining lifespan during which time radiation-related cancer might develop, we also estimated the lifetime risk of developing radiation-related brain cancer for each child in our cohort.

\section{Materials and methods}

Our calculation of dose to the brain was based on estimating the amount of absorbed energy from X-rays within a mathematical model of the brain. The brain model, developed by the Medical Internal Radiation Dose Committee, is a mathematical description of the size, composition, and density of the cranium and brain. For each age $(0,1,5,10$ and 15 years, and adult), Bouchet et al. [4] and Bouchet [5] provide the dimensions and characteristics of the cranium and the brain, which is considered as two half-ellipsoids. The caudal layers of the brain model are truncated to simulate the brain stem and cerebellum. For the purpose of our calculations, the brain was divided into $1-\mathrm{cm}$ thick layers, each composed of $1 \mathrm{~cm}^{3}$ cubic volumes. The radiation dose absorbed within each volume of tissue was estimated based on the attenuation of Xradiation from the surface of the head to the depth of each volume. From those calculations, the approximate spatial pattern of absorbed dose was determined, from which the average dose to the entire brain was estimated (see Fig. 1). Additional details on the dose calculation model will be described in a subsequent publication.

Information necessary for these calculations included the age of the patients (because cranium thickness and density as well as brain size are a function of age), entrance dose (or peak skin dose) and characteristics of the radiation fields (energy and geometry of irradiation). Some of this information was available from a review of the literature. Patient-specific data from the RAD-IR database were also used; these data were stripped of protected health information and the research was conducted with the approval of our Institutional Review Board.

Typical devices used for interventional fluoroscopy procedures have two $\mathrm{C}$-shape arms, each with an X-ray tube and image receptor, that can move and rotate about the patient. The proportion of exposure coming from each Xray tube has been investigated; in our dataset, we estimate that about $60 \%$ of the exposure is from the tube below the patient and $40 \%$ from the lateral tube. The distribution of $\mathrm{X}$-ray energies generated by the fluoroscopy machines is determined by settings of potential (voltage) and filtration. Peak potential is set automatically by modern fluoroscopic machines based on patient thickness and is not recorded. From special measurements conducted on a RANDO phantom, it was assumed that for pediatric examinations, peak potentials would rarely be greater than $90 \mathrm{kVp}$. Based on our review of the literature $[6,7]$, we found that in the range of peak potentials usually involved, the attenuation of the broad X-ray spectrum can be reasonably simulated by a single energy at $30 \mathrm{keV}$.

Information on field size and location in the brain was not available for individual cases. We simulated a variety of fields of a size and orientation characteristic of typical pediatric neuroradiologic examinations. In one scenario we considered the head irradiated by two uniform fields as large as the entire brain, with the radiation directed from the $\mathrm{X}$-ray tube below the table (PA geometry) and from the lateral plane. This extreme case is unlikely. In most clinical procedures, the radiation field is focused on the diseased part of the brain, and the entire brain is not irradiated uniformly throughout the procedure. Examination fields are usually not static; they are moved in real-time to track the movement of the catheter within blood vessels. Taking into account these possibilities, a variety of possible field sizes and orientations were investigated, including large and small static and moving fields as well as combinations.

The absorption of energy decreased with depth in the tissue, resulting in the highest dose either immediately below the point of entrance or where fields overlapped. The average brain dose was determined from the dose received within all of the small volumes defined in the agedependent brain phantom (Fig. 1). Based on the average dose to the brain, the risk of developing brain cancer during

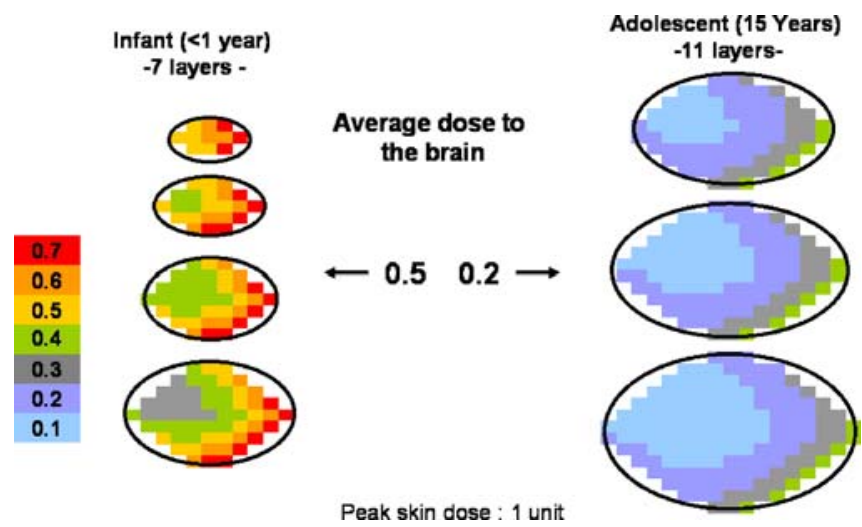

Fig. 2 Comparison of doses from uniform fields 


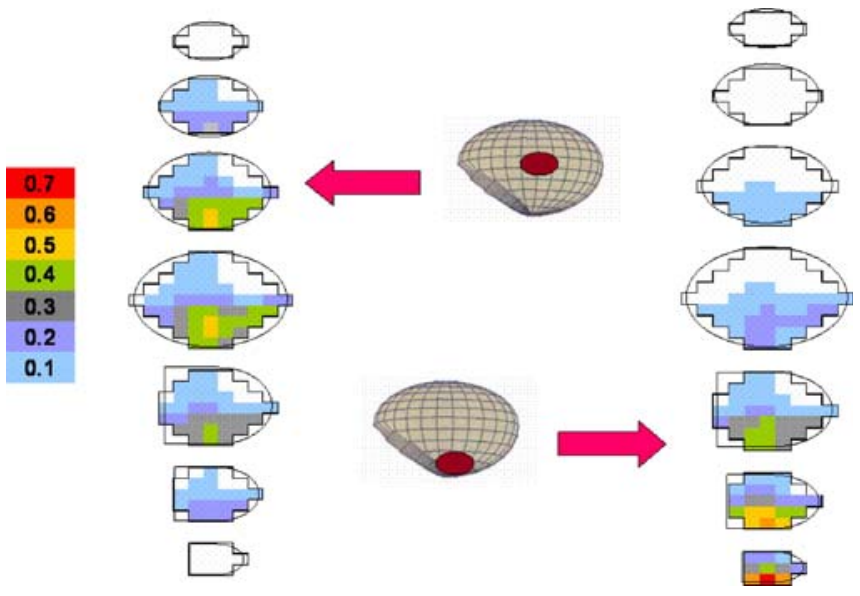

Fig. 3 Dose in the infant's brain-moving fields

the remainder of a normal lifespan was estimated for each case using the Interactive RadioEpidemiological Program [8] developed by the US National Cancer Institute for estimating the probability of cancer causation following radiation exposure. IREP calculates Assigned Share, defined as $\mathrm{AS}=\mathrm{ERR} /(1+\mathrm{ERR})$, for a specific age at cancer diagnosis. For the present paper, age-specific estimates of AS were obtained from IREP for each year of age after exposure and converted to $\mathrm{ERR}=\mathrm{AS} /(1-\mathrm{AS})$, and a summary ERR value was computed as a life-tableweighted average of the age-specific values.

\section{Results}

Irradiation by two large uniform fields

Spatial patterns of absorbed radiation dose in several layers of the brain phantom, from the center slice to the top of the brain, are shown in Fig. 2 for the infant and the adolescent. Dose within each $1 \mathrm{~cm}^{3}$ cube is shown relative to 1 unit peak skin dose. The highest brain dose occurred, in general, where frontal and lateral examination fields overlapped. For the infant, the highest dose in any small volume in the brain reached 0.7 for each unit peak skin dose. For the adolescent, the attenuation by the much thicker cranium had a significant effect. As a result, for 1 unit peak skin dose, the highest dose in any small volume was only 0.4 . When the dose in all of the small volumes of the phantom was considered, the brain dose was 0.5 per unit of peak skin dose for the infant and 0.2 for the adolescent.

This scenario of irradiation of the brain by two large uniform fields during the entire procedure is an unusual case. Radiologists are trained to collimate the fields in order to expose the smallest area necessary.

\section{Irradiation by small moving fields}

Through most of the examination time, the field was collimated on the diseased part of the brain rather than on the entire organ. Figure 3 gives a representation of two possible cases with the pathologic focus at the center of the brain, or at the edge. As shown in Fig. 3 and Table 1, average radiation doses were dramatically reduced when fields were collimated; reductions by about $70 \%$ for the infant and $85 \%$ to $95 \%$ for the adolescent were possible, depending on the location of the pathology within the brain. Note that the dose to small regions of the brain could still be quite high even if the average organ dose was small.

Lifetime risk of developing radiation-related brain cancer

Based on the estimated radiation dose to the brain where the brain is irradiated by two large uniform fields, or by two small fields with the pathologic focus at the center of the brain, the risk of developing radiation-related brain cancer, relative to non-exposed children, was estimated for the least-exposed child in the cohort, a 2-year-old boy, and the most exposed child, a 14-year-old boy (Table 2). For the least-exposed child, the lifetime risk of developing brain cancer compared to a non-exposed child of the same age was estimated to be increased by $2 \%$ to $10 \%$ (a relative risk of 1.02 to 1.10); that is, for children exposed similarly, the number of cases of brain cancer to occur would be increased from 65 per 10,000 (the baseline) [9] to approximately 66 or 71 , depending on the actual conditions of exposure. In contrast, for the most highly exposed child (14 years of age at exposure), the increase in risk ranged from $10 \%$ to $80 \%$, depending on the actual conditions of his exposure, meaning that for each 10,000 children exposed similarly, the number of cases of brain cancer expected to occur would increase from 65 per 10,000 to approximately $71-117$.

\section{Discussion}

Because no data are available on doses received to the brain in children undergoing cerebral embolization procedures, we developed a calculation strategy to estimate the spatial pattern of dose and average dose to the organ. Our calculations were applied to a cohort of 50 children who had been treated for aneurysms, arteriovenous malformations, or tumors in the brain. Because these interventions are uncommon in children, and radiation dose is usually not measured, our cohort likely represents the largest set of data available for which radiation dose and risk can be estimated following such procedures. The main factors

Table 1 Comparison of average dose to the brain for the infant and the adolescent ( 1 unit peak skin dose)

\begin{tabular}{llll}
\hline Age (years) & Large uniform fields & \multicolumn{2}{l}{ Narrow non-uniform fields } \\
\cline { 3 - 4 } & & Diseased part of the brain \\
\cline { 3 - 4 } & & Middle & Bottom \\
\hline$<1$ & 0.52 & 0.15 & 0.12 \\
15 & 0.21 & 0.03 & 0.01 \\
\hline
\end{tabular}


Table 2 Predicted relative risk of brain cancer from radiation received during interventional procedure

\begin{tabular}{llcl}
\hline Field & & $\begin{array}{l}\text { Average dose to Relative } \\
\text { the brain (mGy) }\end{array}$ \\
\hline risk
\end{tabular}

${ }^{a}$ Relative to non-exposed of same age

influencing dose are patient age (age is related to density and thickness of a child's cranium), duration of fluoroscopy, portion of the brain irradiated, and degree of overlap of radiation fields. It is apparent that even if the average organ dose is low, the maximum dose received at localized points can still be high, particularly where fields overlap. As we have discussed, if radiation procedures are conducted with the intent of minimizing unnecessary exposure, i.e., if ALARA principles are followed, the future risk of developing brain cancer is not greatly enhanced compared to the risk experienced by non-exposed children. To better understand the actual doses received and to better estimate the lifetime cancer risks, we recommend that better and more detailed record-keeping of machine parameters and specifics of the exposure conditions become standard procedure.

\section{Conclusion}

Interventional radiology procedures are potentially lifesaving, and it is widely understood that the requirement for these procedures often outweighs considerations for minimizing radiation dose. However, minimizing cancer risk for the future also serves patients. Applying ALARA principles of collimation and shortest possible exposure time are the most effective means to minimize risk of future radiation cancer.

\section{References}

1. Balter S, Schueler BE, Miller DL, et al (2004) Radiation doses in interventional radiology procedures: the RAD-IR study. Part III: dosimetric performance of the interventional fluoroscopy units. J Vasc Interv Radiol 15:919-926

2. Miller DL, Balter S, Cole PE, et al (2003) Radiation doses in interventional radiology procedures: the RAD-IR study. Part I: overall measures of dose. J Vasc Interv Radiol 14:711-727

3. Miller DL, Balter S, Cole PE, et al (2003) Radiation doses in interventional radiology procedures: the RAD-IR study. Part II: skin dose. J Vasc Interv Radiol 14:977-990

4. Bouchet LG, Bolch WE, Weber DA, et al (1999) MIRD pamphlet 15: radionuclide $\mathrm{S}$ values in a revised dosimetric model of the adult head and brain. J Nucl Med 40:62S-101S

5. Bouchet LG (1999) Five pediatric head and brain mathematical models for use in internal dosimetry. J Nucl Med 40:1327-1336

6. Harrison RM (1981) Central-axis depth-dose data for diagnostic radiology. Phys Med Biol 26:657-670

7. Harrison RM (1983) Tissue-air ratios and scatter-air ratios for diagnostic radiology (1-4 mm Al HVL). Phys Med Biol 28:118

8. Land CE, Gilbert E, Smith J, et al (2003) Report of the NCICDC Working Group to revise the 1985 NIH radioepidemiological tables. NIH Publication no. 03-5387. Report available at http://dceg.cancer.gov/docs/Report03.pdf, Interactive RadioEpidemiological Program (IREP) v.5.3. Online software available at http://www.irep.nci.nih.gov/

9. Ries LA, Eisner MP, Kosary CL, et al (2005) SEER Cancer statistics review, 1975-2002. Edwards BK (ed) National Cancer Institute. Bethesda, MD. http://seer.cancer.gov/csr/1975_2002/, based on November 2004 SEER data submission, posted to the SEER web site 